\title{
Simulation of a high-speed train control system based on High Level Architecture and its credibility analysis
}

\author{
Wei ShangGuan, J.-Q. Chen, B. Li, L.-N. Guo, M. Li \& L.-Y. Chen \\ State Key Laboratory of Rail Traffic Control and Safety, \\ School of Electronic and Information Engineering, \\ Beijing Jiaotong University, China
}

\begin{abstract}
The study of the simulation of a high-speed train control system has great significance for the realization of a train control system. This paper studied the basic theory of a high-speed train control system in China. Based on the theory and structure of HLA (High Level Architecture), multi-resolution modelling, simulation real-time management methods and the system architecture of a highspeed train control system simulation was studied systematically, and the simulation result of the on-board vehicle and field centre equipment was shown. With the aim of establishing the credibility of simulation, the methods of VV\&A, qualitative and quantitative RAMS analysis and system fault injection were studied, which improved the credibility of high-speed train control system simulation.
\end{abstract}

Keywords: high-speed train control system, HLA, high level architecture, credibility analysis, multi-resolution modelling, fault injection.

\section{High-speed train control system}

The Chinese railway department makes a set of CTCS standards that are fit for Chinese actual conditions by referring to the ERTMS/ETCS standards.The CTCS standards have made an overall technical program and master plan for the great-leap-forward development of China's railway signalling system. CTCS is classified into five grades, from CTCS-0 to CTCS-4. Its structure is made up of the railway transport management layer, the network transport layer, the ground equipment layer and the on-board equipment layer [1, 2]. 


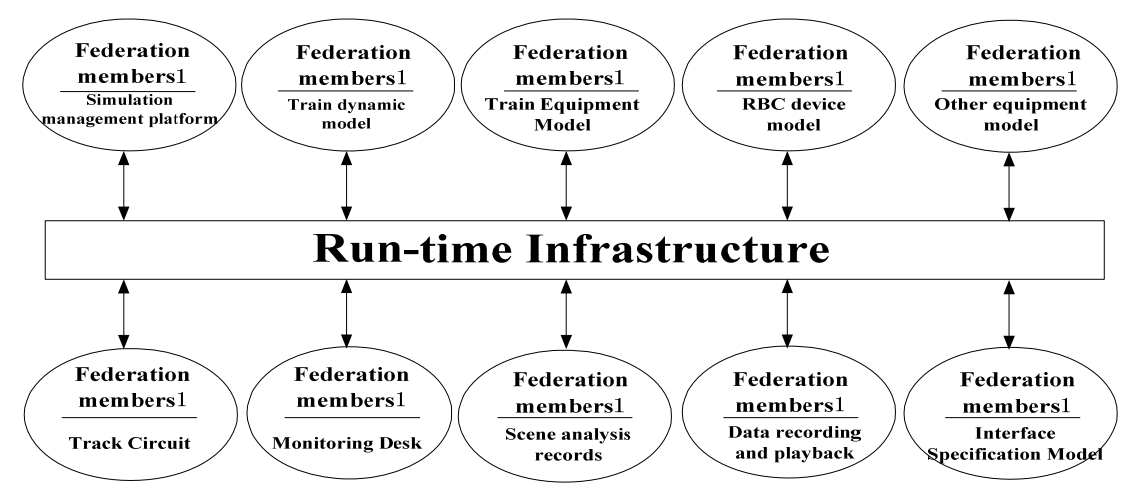

Figure 1: The federal structure of the high-speed train control system's simulation platform.

CTCS is a technical specification classified into grades for different transport requirements to be used to ensure the train's operational safety. We have determined the need to adopt the CTCS-3 train control system, with high reliability and high safety, as the general technical platform to be consulted for the passenger specific line and the Beijing-Shanghai express railway.

\section{High Level Architecture for modelling}

High Level Architecture is a new framework for distributed simulation, which was developed by the U.S. Department of Defense to meet the needs of interconnection and interoperability problems of a variety of simulation systems with multiple-models, which were developed in various fields. In a HLA simulation system, the federate is a distributed simulation system used to achieve a particular simulation purpose. It consists of several interactive federal members. All the applications participating federal running can be called federal members $[3,4]$.

According to the functional analysis of the Simulation Integration Platform for the Train Control System, we built the Simulation system's Federation based on HLA. Federation members are shown in Fig 1.

\section{High-speed train control system simulation system based HLA}

\subsection{Multi-resolution Modelling}

Multi-resolution Modelling is a modelling method that uses multi-precision and multi-level approaches to model a system. A high-speed train control system's Multi-resolution Modelling is defined as: in the process of modelling and simulation, making the details of interaction with different levels of information as the criteria, we use the method with different precision and different levels to 
descript every function of the train control system to improve simulation fidelity or improve simulation efficiency. These precision and levels are defined as the high-speed train control system's modelling resolution [5].

After understanding the basic structure of the high-speed train control system and the division of the train members, to be directed against a different person's focus on different sides of train equipment, such as ground equipment and trainground information exchange processes, we research the establishment of the high-speed train control system's multi-resolution model on the basis of existing research. In this paper, the high-speed train control system is divided into three types of resolution, using details of interaction with different levels of information as the criteria, as shown in Fig 2.

Low-resolution information (the top) is embodied by the train moving, obtaining the speed and location information generally. Medium-resolution information is embodied by exchanging information among members. The establishment of this model is favoured to check whether the information channel is established. Because this model does not involve computing information and access, it does not only effectively reflect the interaction of information, but does easily grasp the overall message. The high-resolution model is embodied by the calculation of the various members of the internal information and access, and this model can be used to test the accuracy of the information.

\subsection{Simulation of real-time management}

How do we realize the data exchange between the model points? We process data exchange with the RTI of the HLA to ensure real-time and reliability. The RTI is assistant software system of the HLA. The basic way to improve network trial-time is to advance the RTI performance [6].

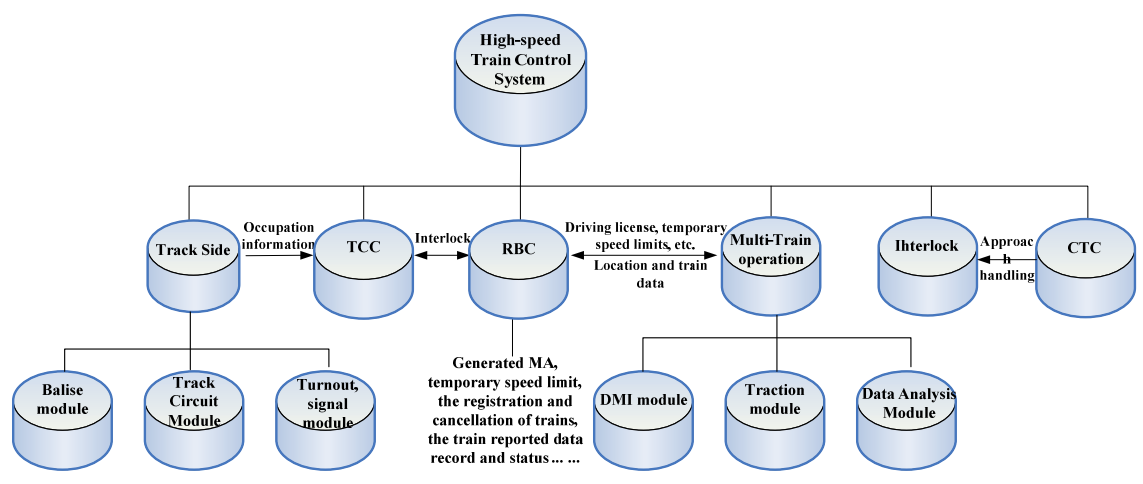

Figure 2: Instantiation of multi-resolution in the high-speed train control system. 


\section{1) With Multithread}

In HLA, the process mode decides the ways that the application process (member) calls the RTI and the RTI call callback function. At the same time, it decides members share the CPU clock with the RTI.

There are three process modes for the RTI: single thread, asynchronous mode and multithread. For single thread mode, the member must use the RTI and call tick() function to complete the callback in a thread. For the synchronous mode, it assists the multithread reentrant. It calls the RTI service with a thread and continuous calls tick() with another thread. Its performance is better than single thread. For the multithread mode, the member does not need to call tick(). Message process and callback could auto complete in the RTI. There is no message starvation state and message obstruction state. Because this makes full use of the CPU clock, it effectively improves network real-time.

2) Further Improvement to the Data Filtering Mechanism

Net data excess leads to network obstruction and delay. HLA provides a data filtering mechanism based on class and value to limit redundant data in the system and reduce system source pressure. The communication mechanism that points to multipoint in distributed interactive simulation applies multicast communication well in a distributed interactive simulation environment, especially combined with the data filtering mechanism, leading to reduced network traffic and simulator point communication load. As a result, it improves the system's scalability.

3) Improve Time Management Algorithm

Data manage (DM) and time manage (TM) are the focus and difficulty points of HLA/RTI. The RTI's time management algorithm used for reference Parallel Discrete Event Simulation (PDES). Its important key is Lower Bound of Time Stab (LBTS). Based on the test data of the network delay aforementioned, we can establish that there is a message transfer delay between nodes, which is caused by time advance. When a member requires the RTI to provide a Time Advance Request (TAR), the RTI provides LBTS to the members who ask for a TAR, according to member's TM mode (time limit and time limited) and their logic time and time lookahead, and reacts to the request. Improving the time management algorithm's key improves the time advance request's reaction. In other words, it is a LBTS algorithm.

4) Improve System Real-time from the Application Level

Considering that the time-delay of the RTI responding to the time advance request from the federate is the main factor in affecting the real time of the system, we can reduce the times taken to request, even coordinating the time advance request without the RTI. In that case, the real-time performance of the system will be significantly improved. Based on such consideration, a new improved strategy is researched in this paper. Experimental studies show that the strategy can effectively reduce the time-delay caused by the RTI and improve the real-time performance of the system.

Time management service is an important aspect that the HLA distinguishes with the DIS system. In addition, it is the problem of bottleneck that affects the real-time performance of HLA. With the problem of bottleneck unsolved, we can 
coordinate the time synchronization between the federate by an application layer without the time management service, and the other services that the RTI provides are still valid.

Fig. 3 shows that the time management mentioned above integrates into the simulation of the high-speed train control system. Through reducing the number of time advances in the application layer, it can save time. The time advance strategy of the RTI itself is achieved by improving the data filtering mechanism, using multi-thread, improving time management algorithms according to the data needs of the application layer.

\subsection{Architecture of high-speed train control system simulation}

Because of the problem that using simulation becomes more and more complicated, it is meaningful to improve the authenticity, timeliness, usability, reusability and interoperability of the simulation of the high-speed train control system with using High Level Architecture (HLA) [7].

The high-speed train control simulation system is divided into the simulation management subsystem and simulation equipment modules, which are put together into the HLA/RTI environment as federations. Fig. 4 shows the architecture of the system.

The simulation management subsystem includes a line database, verify and analysis module and simulation management module. Before the simulation, it can complete the data preparation and configure other federations by the HLA/RTI underlying environment. During the simulation process, it can start the simulation and issue orders. After simulation, it can verify and analyze the data and evaluate the entire platform.

Simulation equipment modules include the interlocking module, CTC module, train control centre, etc. As a result of HLA, we can separate the bottom environment and the application layer, and each simulation module can be individually designed according to different needs and concerns with different algorithm models.

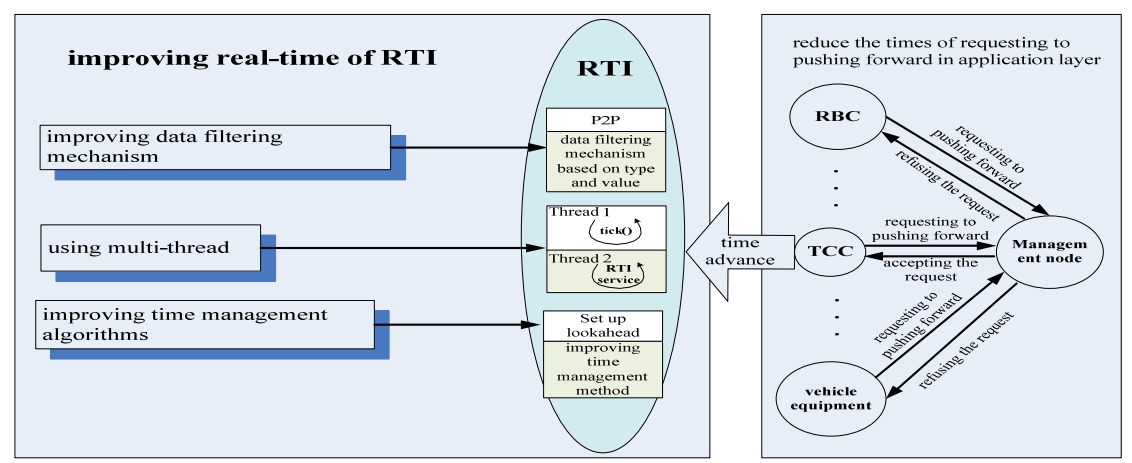

Figure 3: Time management module plan. 


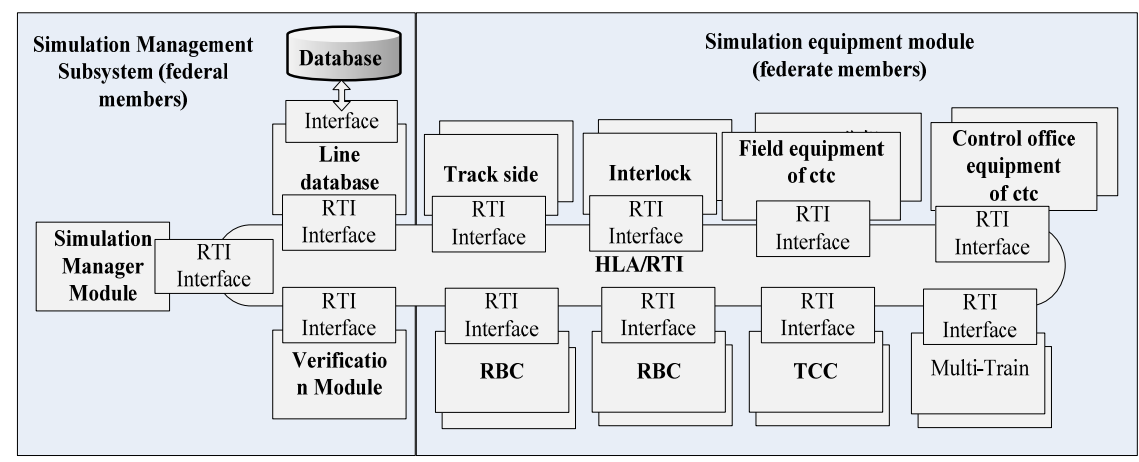

Figure 4: Architecture of the high-speed train control system simulation based on HLA.
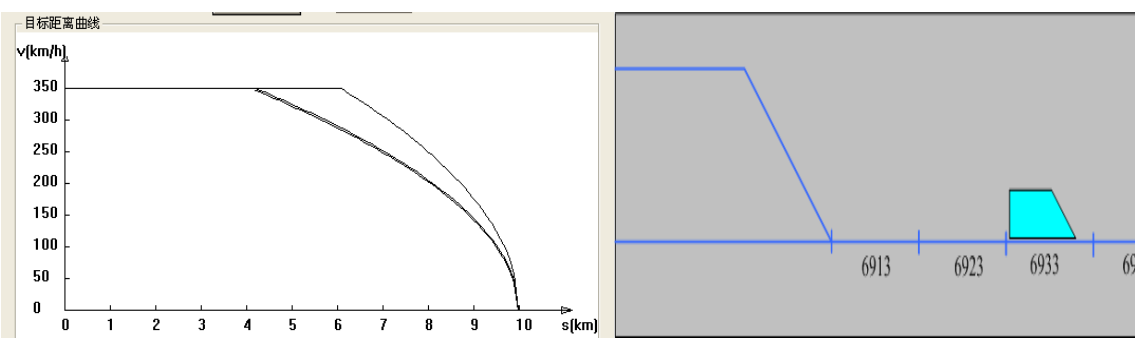

Figure 5: On-board equipment speed control modelling.

\subsection{Simulations}

The on-board equipment and ground equipment are achieved based on the HLA multi-resolution modelling method and time management strategies in this paper. Fig. 5 shows the simulation of vehicle equipment. Fig. 6 shows the simulation of the $3 \mathrm{D}$ view and the DMI module simulation. Fig. 7 shows the ground equipment, CTC module and interlocking module simulation.

\section{Analysis of credibility for simulation}

After the high-speed train control system simulation is built, the analysis of credibility for the simulation system is needed based on the simulation environment.

\subsection{Loop simulation model and analysis of $\mathrm{VV \& A}$}

At present, there are two categories for train control system simulation, one is total digital simulation, and the other is loop simulation. In the design and assessment of a complex train control system, the latter is more beneficial, which 
can not only descript the process of train running, but also can put the real equipments into the simulation. In order to ensure the credibility of the train control system, the VV\&A workflow is used, as shown in Fig. 8.
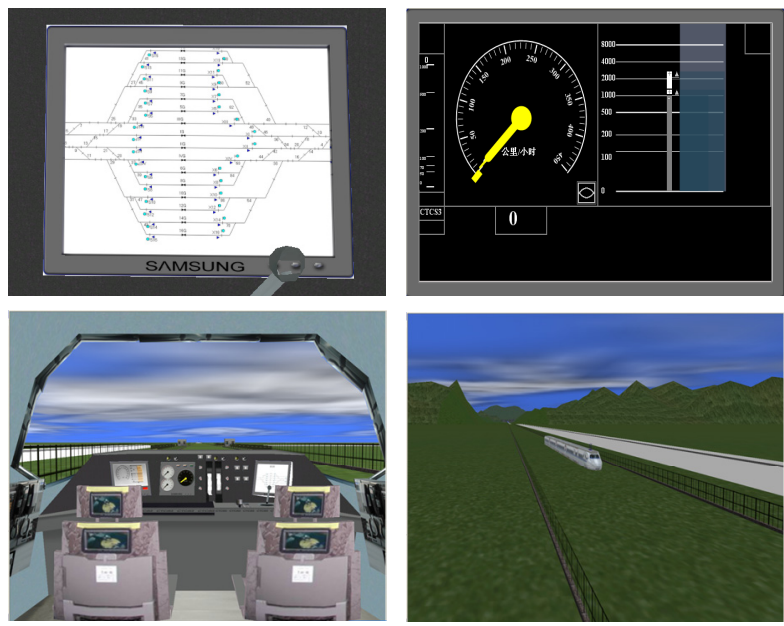

Figure 6: On-board view and DMI.
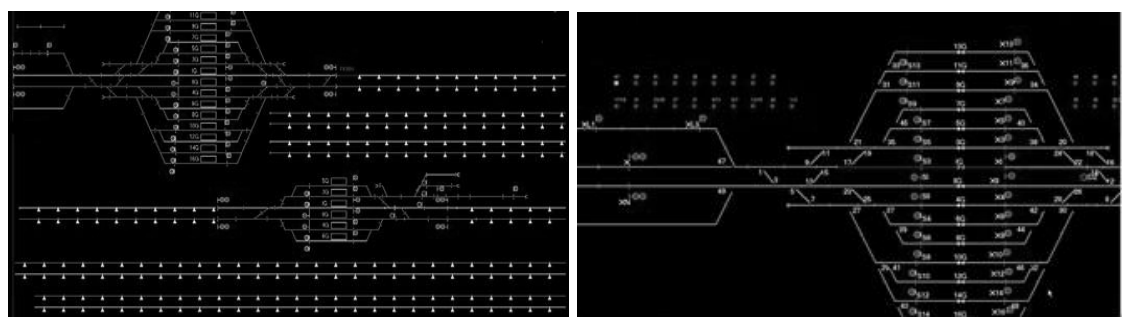

Figure 7: Simulation of CTC and interlocking.

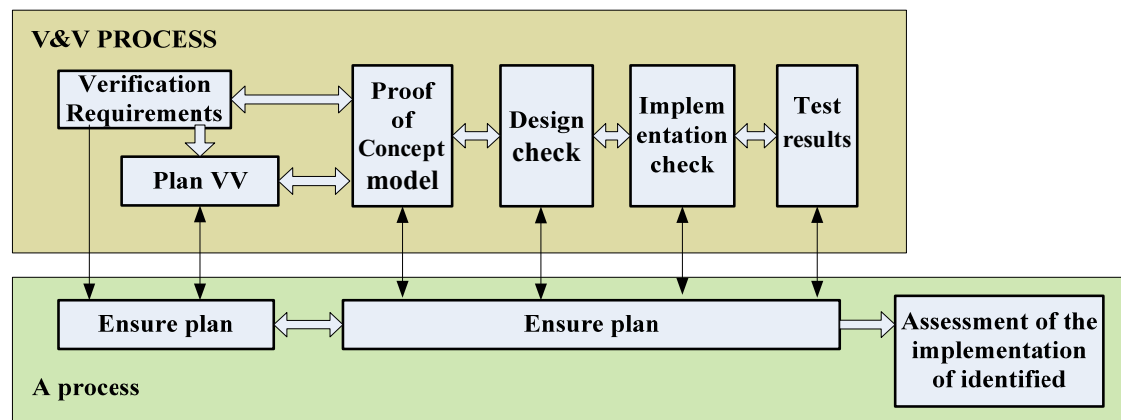

Figure 8: VV\&A flowchart of simulation. 
For the problems of the credibility of complex system, such as the high-speed train control system, after analysis, synthesis, determination and formation of function modules related to the credibility problems, and according to the relationship and importance, the function structure is shown in Fig.9.

There is only one factor in the highest layer of the complex simulation system that is credible for the purpose of solving problems, which is also the overall goal, so this layer is also called the target layer.

$>\quad$ The middle layer represents the intermediate links that adopt and implement the programs used to achieve the overall goal, which is generally called the strategy layer, constraint layer or rule layer. Through the analogy, according to the characteristics of the simulation system, the middle layer is designed to the subsystem's credibility and function module's credibility, such as the subsystem's credibility $(1,2, \ldots, m)$ and function credibility $(1,2, \ldots, n)$, which can be further broken down based on the actual system.

$>\quad$ The bottom layer is the credibility of the sub-function modules. These subfunction modules are characterized as being more independent and credible and they are easily measured, providing more detail than the middle layer.

\subsection{High-speed train control system for qualitative and quantitative analysis of RAMS}

RAMS is the reliability, availability, maintainability, and safety of the short.

RAMS in the train control system is subject to the following aspects: fault source triggered within the system, failure source introduced in the system operation phase, failure source introduced in system maintenance activities. RAMS and their interrelations in the train control system are shown in Fig.10.

In order to achieve quantitative analysis of the RAMS in the train control system, they need to define a quantitative expression. The tandem structure of

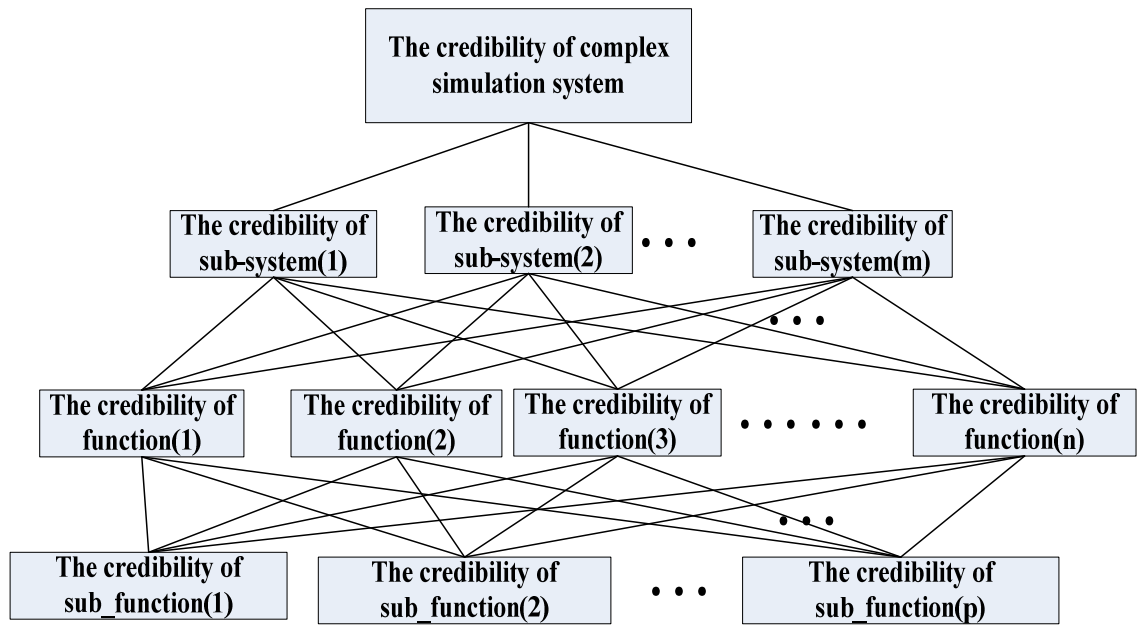

Figure 9: $\quad$ Simulation system function structures chart. 


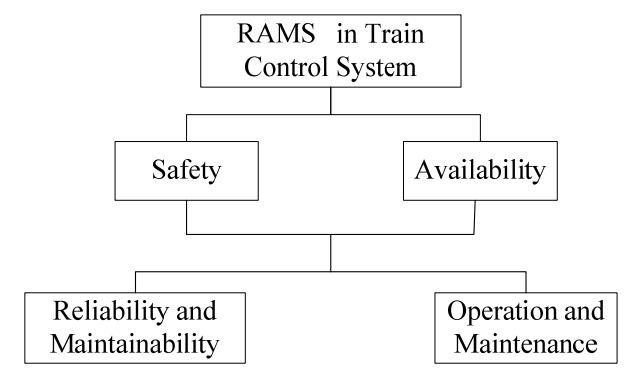

Figure 10: $\quad$ RAMS and their interrelations.

the train control system usually use means time between failures (MTBF) to measure each subsystem and the entire subsystem reliability [8].

$$
M T B F_{\text {System }}=\frac{1}{\sum_{i=1}^{n} q_{i} \lambda_{i}}
$$

The train control system's maintainability is expressed by the average time required to identify the failure and return to normal state and maintenance convenience. Usually we use mean time to repair (MTTR) to measure this, expressed by the formula:

$$
\operatorname{MTTR}_{\text {System }}=\frac{\sum_{i=1}^{n} q_{i} \lambda_{i} M T T R_{i}}{\sum_{j=1}^{n} q_{j} \lambda_{j}}
$$

where $q_{i}$ is the number for $i$ device and $\lambda_{i}$ is the failure rate for $i$ device.

In the quantitative analysis of the system RAMS, the Markov method has powerful functions. It can fully reflect impact from the system testing and maintenance and the time-varying characteristics of real-time response systems. The Markov method can also be calculated using a number of different RAMS indicators, such as the system reliability within a certain time period, the availability of a moment and the MTTF.

For the general mathematical model of the Markov chain, suppose $\{X(n), n=0,1,2, \ldots \ldots\}$ is a value in the $E=\{0,1,2, \ldots \ldots\}$ or $E=\{0,1,2, \ldots \ldots, N\}$ on a random process, the former expressed as an unlimited number of states. In the latter case, it is expressed as a limited number of state spaces. The following formula will be used for the definition of Markov chains.

Suppose $\{X(n), n=0,1,2, \ldots \ldots\}$ random sequence of discrete state space for $E$. If for any $m$ non-negative integer $n_{1}, n_{2}, \ldots \ldots, n_{m}\left(0 \leq n_{1} \leq n_{2} \leq \ldots \ldots \leq n_{m}\right)$ and any natural number $k$, and arbitrary $\mathrm{T}$, to satisfy:

$$
\begin{aligned}
P\left\{X\left(n_{m}+k\right)\right. & \left.=j \mid X\left(n_{1}\right)=i_{1}, X\left(n_{2}\right)=i_{2}, \ldots \ldots X\left(n_{m}\right)=i_{m}\right\} \\
& =P\left\{X\left(n_{m}+k\right)=j \mid X\left(n_{m}\right)=i_{m}\right\}
\end{aligned}
$$

This illustrates an important property of the Markov process: it has a no aftereffect nature, which is also known as non-memory. The RAMS analysis flowchart based on the Markov chain is shown in Fig. 11. 


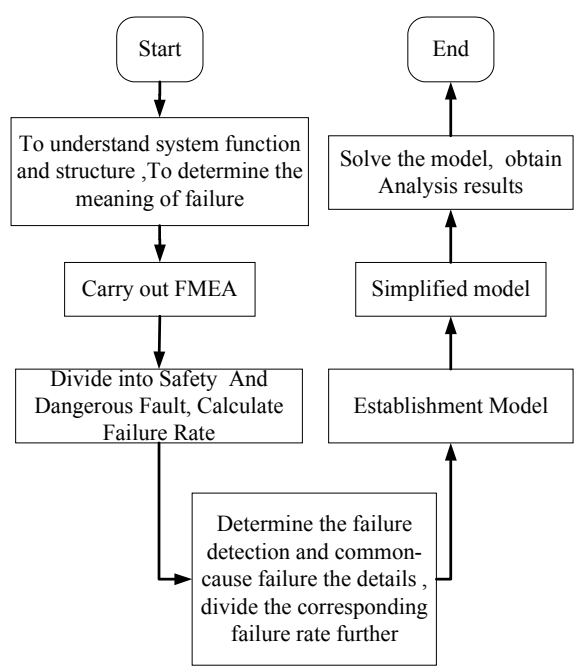

Figure 11: RAMS analysis flowchart based on the Markov chain.

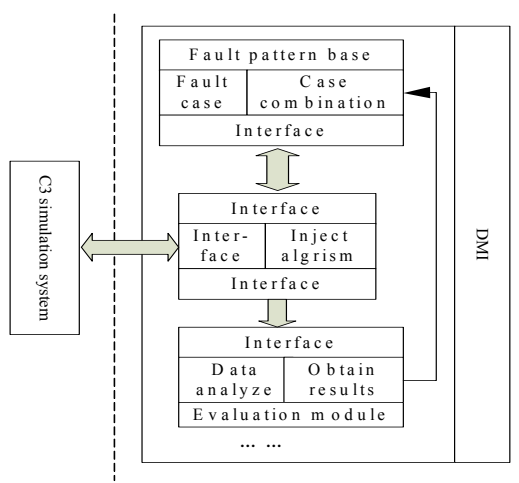

Figure 12: General structure of the software fault injection system.

Through the merger of the state, the Markov model can be greatly simplified. In addition, there are a number of other Markov model simplification techniques, for example, the system decomposition and model compression. In the case that the system is relatively large and complex, one can use these technologies.

\subsection{Realization of the fault injection system}

The general structure of the software fault injection system is shown in Fig. 12. This system has three modules, a fault injection module, fault pattern base and evaluation module [9].

The fault pattern base plays an important part in fault injection. A good fault pattern base could improve fault injection quality efficiently. The fault patter base is composed of the fault case coding module, fault tree module, automatic evaluation module and evaluation result module, as shown in Fig. 13. 


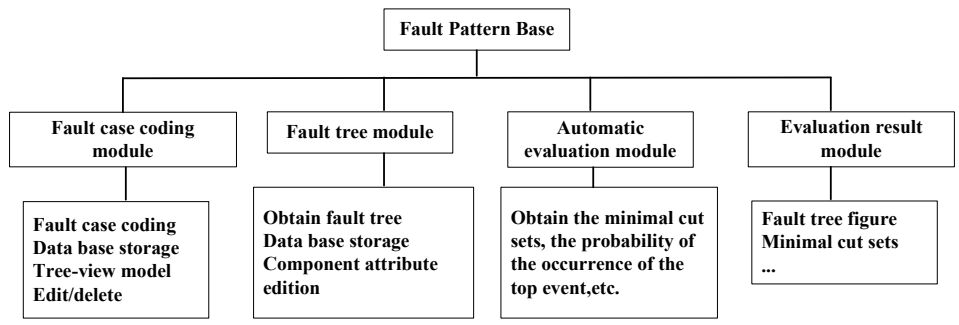

Figure 13: Fault pattern base function module.

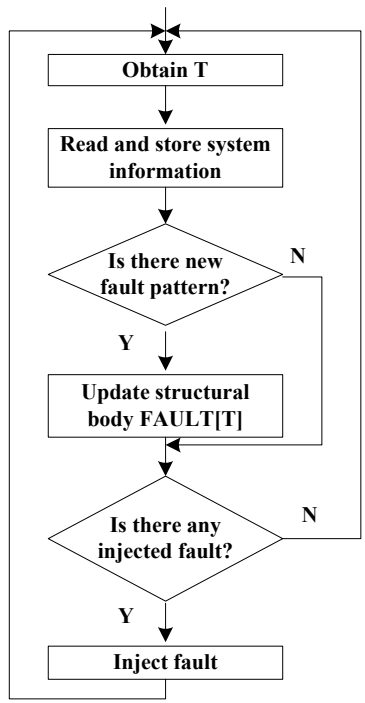

Figure 14: Injection algorism.

The fault injection module finishes one round of fault injection in the following steps: monitor simulation system, collect and transmit operation data, read fault pattern, inject fault, stop injection. The injection algorism injects the structural body, which is coded already, into the simulation system [10]. The process of this algorism is: as soon as the simulation system time is obtained, update the fault pattern structural body; according to the structural body, the fault inject place and fault data are obtained; intercept transmission data and inject fault data.

The fault injection module finishes one round of fault injections in the following steps: monitor the simulation system, collect and transmit operation data, read the fault pattern, inject the fault, stop the injection. The injection algorism injects the structural body, which is coded already, into the simulation system. The process of this algorism is: as soon as the simulation system time is obtained, update the fault pattern structural body; according to the structural body, the fault inject place and fault data are obtained; intercept transmission data and inject the fault data. A flowchart of the injection algorism is shown in Fig. 14. 


\section{Conclusion}

This paper has made a design of a high-speed train control system from a multiresolution model to reliability, analyzing step by step the needs of the high-speed train control system, and HLA is used as the simulation supporting environment. The HLA environment can meet the distributed requirement of the high-speed train control system; Improved RTI time management can meet the requirement of real-time. The high level architecture can show the interaction between modules and the data interfaces, while the multi-resolution can build the modules based on the different concerns. Making use of the advantages of the software simulation system, fault injection is used to inject to the system to get the information caused by the fault; the Markov chain method is used to achieve the qualitative and quantitative analysis of RAMS, while the analysis of VV\&A provides the basis for performance to improve and optimize the system design and confirm the system capability. Furthermore, the methods researched in this paper can be used to analyze effectively the high-speed train control system.

\section{Acknowledgements}

This research work was supported by the Key Program of the National Natural Science Foundation of China (No.60736047, 60870016), Independence Research Task of State Key Laboratory of Rail Traffic Control and Safety (RCS2009ZT013), Technological Research and Development Programs of the Ministry of Railways (No. Z2009-059), Science and Technology Foundation of BJTU (No.2008RC023) and Fundamental Research Funds for the Central Universities (No. 2009JBM005).

R.B.G. thanks Pro. Cai and Dr. Wang who had devoted their attention to my study and guided the right research direction; thanks for my team partners, they have given me a great deal of instructive advice on my research; and thanks for my family, my family's self-giving love is my most important power; thanks for everybody who has ever helped me.

\section{References}

[1] Beijing Railway Administration. CTC-2 train control system used in maintenance [M]. Beijing: Chinese Railway Press. 2007.

[2] Xu Xiaoming, Yuan Xiange, Li Ping. Train operation control system controlling ground equipment books column [M]. Beijing: Chinese Railway Press. 2007.

[3] Zhang Xuguang. CTCS-3 Train Control System Technology Innovation [M] Program of Transportation. 2008.3

[4] Qin Jiandong, Yan Changfeng, Wangdi. Collaborative ship defense simulation system based HLA and UML [J]. Journal of Wuhan University of Technology,2008,30(2):261-264

[5] Liu Baohong. Multi-resolution Modelling Research and development [J]. System Simulation, 2004, 16(6):1150-1154 
[6] Rachel F.M, Cuganasca P.S. Objected-oriented approach for automatic train operation control systems [C]. Computers in Railways IX Proceedings, Wit Press, 2004:421-430

[7] Pataricza I, Majzik G, Huszerl Gy. Várna. UML-based Design and Formal Analysis of a Safety-Critical Railway Control Software Module In G. Tarnai and E. Schnieder (eds.): Formal Methods for Railway Operation and Control Systems (Proceedings of Symposium FORMS-2003, Budapest, Hungary, May 15-16), L. Harmattan, Budapest, 2003:125-132.

[8] Decknatel G, Slovak R, Schnieder E. Definition of a Type of ContinuousDiscrete High-Level Petri Nets and Its Application to the Performance Analysis of Train Protection Systems. In Engell S, Frehse, G, Schnieder E, Hrsg. Modelling, Analysis, and Design of Hybrid Systems, Springer, Berlin, 2002:355-367

[9] Decknatel G. Modelling Train Movement with Hybrid Petri Nets. FME Rail Workshop, Stockholm, 1999, 99(5):11-12.

[10] Adelantado M., Bonnet S. and Siron P. Multi-resolution Modelling and Simulation with the High Level Architecture. Proceedings ESS'2000, 12th European Simulation Symposium, 2000:1-6 